\title{
EXTREMAL MULTILINEAR FORMS ON BANACH SPACES
}

\section{SARANTOPOULOS}

Abstract. Suppose that $L$ is a continuous symmetric $m$-linear form defined on a complex Banach space $E$, and $\hat{L}$ is the associated homogeneous polynomial. If

$$
\|L\|=\left(m^{m} / m !\right)\|\hat{L}\|,
$$

we prove that $E$ contains an almost isometric copy of $l_{m}^{1}$. In particular if $E$ is an $m$-dimensional space, then $E$ is isometrically isomorphic to $l_{m}^{1}$. We also prove that the only examples of such extremal $L$ which achieve their norm are suitable "extensions" of a known example given by Nachbin.

Throughout this paper $K$ denotes either the complex field $\mathbf{C}$ or the real field $\mathbf{R}$. Given any index set $\Gamma$ we denote by $l^{1}(\Gamma)$ the collection of all $K$-valued families $x=\left(x_{i}\right)$ such that

$$
\|x\|:=\sum_{\Gamma}\left|x_{i}\right|
$$

is finite. If $\Gamma$ is the set of positive integers we denote $l^{1}(\Gamma)$ by $l^{1}$, while if $\Gamma=\{1, \ldots, n\}$, where $n$ is a positive integer, we denote $l^{1}(\Gamma)$ by $l_{n}^{1}$. If $E$ is a vector space over the field $K$ we write $E^{m}$ for the product $E \times \cdots \times E$ with $m$-factors. An $m$-linear form $L: E^{m} \rightarrow K$ is said to be symmetric if

$$
L\left(x_{1}, \ldots, x_{m}\right)=L\left(x_{\sigma(1)}, \ldots, x_{\sigma(m)}\right)
$$

for any $x_{1}, \ldots, x_{m}$ in $E$ and any permutation $\sigma$ of the first $m$ natural numbers.

If $E$ is a normed space over $K$, we denote by $\mathscr{L}_{m}^{s}(E, K)$ the space of all continuous symmetric $m$-linear forms $L: E^{m} \rightarrow K$. A mapping $P: E \rightarrow K$ is said to be a homogeneous polynomial of degree $m$ if $P=\hat{L}$ for some $L \in \mathscr{L}_{m}^{s}(E, K)$, where $\hat{L}$ is defined by

$$
\hat{L}(x)=L(x, \ldots, x) .
$$

If $L \in \mathscr{L}_{m}^{s}(E, K)$ we define the norms of $\hat{L}$ and $L$ by

$$
\begin{aligned}
& \|\hat{L}\|=\sup \{|\hat{L}(x)|:\|x\| \leqslant 1\}, \\
& \|L\|=\sup \left\{\left|L\left(x_{1}, \ldots, x_{m}\right)\right|:\left\|x_{i}\right\| \leqslant 1(i=1, \ldots, m)\right\} .
\end{aligned}
$$

Mazur and Orlicz investigated relationships between $\|L\|$ and $\|\hat{L}\|$, and in the Scottish Book [8] conjectured that for any normed space $E$

$$
K(m, E) \leqslant m^{m} / m !
$$

Received by the editors December 3, 1985.

1980 Mathematics Subject Classification (1985 Revision). Primary 46B99.

C1987 American Mathematical Society $0002-9939 / 87 \$ 1.00+\$ .25$ per page 
where

$$
K(m, E)=\min \left\{M:\|L\| \leqslant M\|\hat{L}\| \text { for every } L \in \mathscr{L}_{m}^{s}(E, K)\right\} .
$$

(We shall write $R(m, E), C(m, E)$ instead of $K(m, E)$ if the normed space is real, complex respectively.) This conjecture was subsequently proved by Martin [4]. Notice that the constant $\mathrm{m}^{\mathrm{m}} / \mathrm{m}$ ! depends only on the integer $m$ and not on the normed space.

Let $\Phi \in \mathscr{L}_{m}^{s}\left(l_{m}^{1}, K\right)$ be defined by

$$
m ! \Phi\left(x^{1}, \ldots, x^{m}\right)=\sum_{\sigma \in S_{m}} x_{1}^{\sigma(1)} \cdots x_{m}^{\sigma(m)},
$$

where $x^{i}=\left(x_{n}^{i}\right)_{n=1}^{m}$ for $i=1, \ldots, m$ and $S_{m}$ is the set of permutations of the first $m$-natural numbers. It can be shown, see [1, p. 45], that for this special $\Phi$ we have

$$
\|\Phi\|=\left(m^{m} / m !\right)\|\hat{\Phi}\| \text {. }
$$

Hence the universal constant $\mathrm{m}^{m} / m$ ! is the best possible. In the following, $E$ denotes a complex normed space, unless otherwise specified. The distance between two Banach spaces $X$ and $Y$ is defined by

$$
d(X, Y)=\inf \left\{\|T\|\left\|T^{-1}\right\|: T \text { is a linear isomorphism from } X \text { onto } Y\right\} .
$$

We say that $X$ contains an almost isometric copy of $Y$ if for any $\varepsilon>0$, there exists a subspace $Z$ of $X$ such that $d(Y, Z)<1+\varepsilon$. (In other words $Y$ is $(1+\varepsilon)$-isomorphic to $Z$.)

We now come to our first main result.

THEOREM 1. Suppose that $C(m, E)=m^{m} / m$ ! for some positive integer $m$. Then $E$ contains an almost isometric copy of $l_{m}^{1}$.

To prove Theorem 1 we need a polarization formula. We define the function $s_{n, \beta}$ on $[0,1]$ by

$$
s_{n, \beta}(t)=e^{i \beta_{n}} r_{n}(t), \quad n=1,2, \ldots,
$$

where $\beta=\left(\beta_{1}, \beta_{2}, \ldots\right)$ is a sequence of real numbers and $r_{n}$ is the $n$th Rademacher function. The functions $\left\{s_{n, \beta}\right\}_{n=1}^{\infty}$ form an orthonormal set in $L^{2}([0,1], d t)$, where $d t$ denotes Lebesgue measure. We omit the proof of the following lemma which is similar to the proof of Lemma 2 in [5].

LEMma 1 (Polarization fORMUla). If $E$ is a complex vector space, if $L$ is a symmetric m-linear form, and if $x_{1}, \ldots, x_{m}$ belong to $E$, then for any $\beta=\left(\beta_{1}, \ldots, \beta_{m}\right)$

$$
\begin{aligned}
& m ! L\left(x_{1}, \ldots, x_{m}\right) \\
& \quad=e^{-2 i\left(\beta_{1}+\cdots+\beta_{m}\right)} \int_{0}^{1} s_{1, \beta}(t) \cdots s_{m, \beta}(t) \hat{L}\left(s_{1, \beta}(t) x_{1}+\cdots+s_{m, \beta}(t) x_{m}\right) d t .
\end{aligned}
$$

Proof of Theorem 1. For given $\varepsilon>0,0<\varepsilon<1$, set

$$
\varepsilon_{1}=\left(m^{m} / 2^{m}\right)\left[1-(1-\varepsilon / 2 m)^{m}\right]
$$


where $m$ is a positive integer. Since $C(m, E)=m^{m} / m$ ! there exists an $L \in \mathscr{L}_{m}^{s}(E, \mathbf{C})$ and unit vectors $x_{1}, \ldots, x_{m}$ in $E$ such that

$$
\left|L\left(x_{1}, \ldots, x_{m}\right)\right| \geqslant\left(\left(m^{m}-\varepsilon_{1}\right) / m !\right)\|\hat{L}\| .
$$

If $a_{1}, \ldots, a_{m}$ are any complex numbers, we can assume that

$$
\left|a_{1}\right|=\max \left\{\left|a_{i}\right|: i=1, \ldots, m\right\} \text {. }
$$

Suppose that $a_{k}=\left|a_{k}\right| e^{i \alpha_{k}}, k=1, \ldots, m$, and put $\beta_{j}=\alpha_{j}-\alpha_{1}, j=2, \ldots, m$. Then

$$
\begin{aligned}
\sum_{k=1}^{m}\left|a_{k}\right| \geqslant & \left\|a_{1} x_{1}+a_{2} x_{2}+\cdots+a_{m} x_{m}\right\| \\
= & \left\|\left|a_{1}\right| x_{1}+\left|a_{2}\right| e^{i \beta_{2}} x_{2}+\cdots+\left|a_{m}\right| e^{i \beta_{m}} x_{m}\right\| \\
= & \left\|\left|a_{1}\right|\left(x_{1}+e^{i \beta_{2}} x_{2}+\cdots+e^{i \beta_{m}} x_{m}\right)+e^{i \beta_{2}} x_{2}\left(\left|a_{2}\right|-\left|a_{1}\right|\right)\right\| \\
& +\cdots+e^{i \beta_{m}} x_{m}\left(\left|a_{m}\right|-\left|a_{1}\right|\right) \| \\
\geqslant & +a_{1}\left|\left\|x_{1}+e^{i \beta_{2}} x_{2}+\cdots+e^{i \beta_{m}} x_{m}\right\|+\sum_{k=2}^{m}\right| a_{k}|-(m-1)| a_{1} \mid .
\end{aligned}
$$

So if we can prove that

$$
\left\|x_{1}+e^{i \beta_{2}} x_{2}+\cdots+e^{i \beta_{m}} x_{m}\right\| \geqslant m-\varepsilon / 2
$$

we shall get

$$
(1-\varepsilon / 2) \sum_{k=1}^{m}\left|a_{k}\right| \leqslant\left\|a_{1} x_{1}+a_{2} x_{2}+\cdots+a_{m} x_{m}\right\| \leqslant \sum_{k=1}^{m}\left|a_{k}\right| .
$$

Thus $\operatorname{span}\left\{x_{1}, \ldots, x_{m}\right\}$ will be $(1+\varepsilon)$-isomorphic to $l_{m}^{1}$ and the theorem will follow.

To prove (4) observe that (3) and the polarization formula (2), with $\beta_{1}=0$, imply

$$
\sum_{\varepsilon_{i}= \pm 1}\left\|\varepsilon_{1} x_{1}+\varepsilon_{2} e^{i \beta_{2}} x_{2}+\cdots+\varepsilon_{m} e^{i \beta_{m}} x_{m}\right\|^{m} \geqslant\left(m^{m}-\varepsilon_{1}\right) 2^{m} .
$$

Now from this last inequality we have

$$
\left\|x_{1}+e^{i \beta_{2}} x_{2}+\cdots+e^{i \beta_{m}} x_{m}\right\|^{m}+\left(2^{m}-1\right) m^{m} \geqslant\left(m^{m}-\varepsilon_{1}\right) 2^{m}
$$

and this proves (4).

From Theorem 1 we conclude that if $C(m, E)=m^{m} / m$ ! for every $m$, then $E$ contains uniformly isomorphic copies of $l_{m}^{1}$ for all $m$. So if $E$ is a Banach space, then $E$ has no type $p>1$, since if it did (see [6]) it could not contain uniformly isomorphic copies of $l_{m}^{1}$ for all $m$. (For the definition of type $p$, see [3, p. 72].)

Notice that the condition that $E$ should contain an almost isometric copy of $l_{m}^{1}$ does not always imply that $C(m, E)=m^{m} / m$ !. To see this consider the complex Banach space $l^{\infty}$. This is the space of all bounded complex-valued sequences $x=\left(x_{i}\right)$ under the norm

$$
\|x\|_{\infty}=\sup \left\{\left|x_{i}\right|: i \in \mathbf{N}\right\}
$$


We know [3, p. 73] that $l^{\infty}$ is not of type $p$ for any $p>1$ and so by Lemma 1.e.4 of [3], $l^{\infty}$ contains almost isometric copies of $l_{m}^{1}$ for every $m$. However

$$
C\left(m, l^{\infty}\right) \leqslant m^{m / 2}(m+1)^{(m+1) / 2} / 2^{m} m !<m^{m} / m ! .
$$

This was established by Harris [2, p. 154], see also [7].

Our second main result concerns norm-achieving extremal multilinear forms.

When $E$ is a Banach space with $C(m, E)=m^{m} / m$ ! we say that $L \in \mathscr{L}_{m}^{s}(E, \mathbf{C})$ is extremal if $\|L\|=\left(\mathrm{m}^{m} / m !\right)\|\hat{L}\|$. We shall show that the only examples of such extremal $L$ which achieve their norm are suitable "extensions" of the canonical example (1).

Given a positive integer $m$, let $n_{1}, \ldots, n_{k}$ be nonnegative integers with $n_{1}$ $+\cdots+n_{k}=m$. If $L \in \mathscr{L}_{m}^{s}(E, \mathbf{C})$, we write $L\left(x_{1}^{n_{1}} \cdots x_{k}^{n_{k}}\right)$ for $L\left(x_{1}, \ldots, x_{1}, \ldots, x_{k}, \ldots, x_{k}\right)$, where $x_{1}$ appears $n_{1}$ times, $x_{2}$ appears $n_{2}$ times, and so on.

TheOREM 2. Let $E$ be a Banach space and let $L \in \mathscr{L}_{m}^{s}(E, \mathrm{C})$ satisfy $\|L\|=$ $\left(m^{m} / m !\right)\|\hat{L}\|$. If $L$ achieves its norm at $\left(x_{1}, \ldots, x_{m}\right) \in E^{m}$, where $x_{1}, \ldots, x_{m}$ are unit vectors in $E$, then

(a) $\hat{L}$ achieves its norm, and

(b) $\hat{L}\left(x_{1}\right)=\cdots=\hat{L}\left(x_{m}\right)=0$.

This theorem is an immediate consequence of the following more general result.

THEOREM 2'. Let $E$ be a Banach space and let $L \in \mathscr{L}_{m}^{s}(E, \mathrm{C})$ satisfy $\|L\|=$ $\left(m^{m} / m !\right)\|\hat{L}\|$. Then the following are equivalent: E.

(i) L achieves its norm at $\left(x_{1}, \ldots, x_{m}\right) \in E^{m}$, where $x_{1}, \ldots, x_{m}$ are unit vectors in

(ii)(a) $\hat{L}$ achieves its norm at the points $\left(e^{i \theta_{1}} x_{1}+\cdots+e^{i \theta_{m}} x_{m}\right) / m$ for all choices of real numbers $\theta_{1}, \ldots, \theta_{m}$, and

(b) $L\left(x_{1}^{n_{1}} \ldots x_{m}^{n_{m}}\right)=0$ for all m-tuples $\left(n_{1}, \ldots, n_{m}\right)$ of nonnegative integers, at least one of which is greater than 1 , satisfying $n_{1}+\cdots+n_{m}=m$.

(iii)(a) $\hat{L}$ achieves its norm at the point $\left(e^{i \theta_{1}} x_{1}+\cdots+e^{i \theta_{m}} x_{m}\right) / m$ for some choice of real numbers $\theta_{1}, \ldots, \theta_{m}$, and

(b) $L\left(x_{1}^{n_{1}} \ldots x_{m}^{n_{m}}\right)=0$ for all m-tuples $\left(n_{1}, \ldots, n_{m}\right)$ of nonnegative integers, at least one of which is greater than 1 , satisfying $n_{1}+\cdots+n_{m}=m$.

Proof. If (i) holds, then

$$
\|L\|=\left|L\left(x_{1}, \ldots, x_{m}\right)\right|=\left(m^{m} / m !\right)\|\hat{L}\| .
$$

We will prove that (5) implies (ii). Let $T^{m}$ be the $m$-fold product of the circle group and let $\lambda$ be Haar measure on $T^{m}$. Thus $d \lambda(\theta)=(1 / 2 \pi)^{m} d \theta_{1} \cdots d \theta_{m}$ and we can show easily that the following polarization formula holds:

$$
m ! L\left(x_{1}, \ldots, x_{m}\right)=\int_{T^{m}} e^{-i \theta_{1}} \cdots e^{-i \theta_{m}} \hat{L}\left(\sum_{j=1}^{m} x_{j} e^{i \theta_{j}}\right) d \lambda(\theta) .
$$


Now from (5) and (6) we get

$$
\begin{aligned}
\left(m^{m} / m !\right)\|\hat{L}\| & =\|L\|=\left|L\left(x_{1}, \ldots, x_{m}\right)\right| \\
& =(1 / m !)\left|\int_{T^{m}} e^{-i \theta_{1}} \ldots e^{-i \theta_{m}} \hat{L}\left(\sum_{j=1}^{m} x_{j} e^{i \theta_{j}}\right) d \lambda(\theta)\right| \\
& \leqslant(1 / m !) \int_{T^{m}}\left|\hat{L}\left(\sum_{j=1}^{m} x_{j} e^{i \theta_{j}}\right)\right| d \lambda(\theta) \\
& \leqslant(1 / m !)\|\hat{L}\| \int_{T^{m}}\left\|\sum_{j=1}^{m} x_{j} e^{i \theta_{j}}\right\|^{m} d \lambda(\theta) \leqslant\left(m^{m} / m !\right)\|\hat{L}\| .
\end{aligned}
$$

So we have

$$
\begin{aligned}
& \left|\hat{L}\left(\sum_{j=1}^{m} x_{j} e^{i \theta_{j}}\right)\right|=\|\hat{L}\|\left\|\sum_{j=1}^{m} x_{j} e^{i \theta_{j}}\right\|^{m}, \\
& \left\|e^{i \theta_{1}} x_{1}+\cdots+e^{i \theta_{m}} x_{m}\right\|=m
\end{aligned}
$$

for all choices of real numbers $\theta_{1}, \ldots, \theta_{m}$. Thus

$$
\left|\hat{L}\left(\left(e^{i \theta_{1}} x_{1}+\cdots+e^{i \theta_{m}} x_{m}\right) / m\right)\right|=\|\hat{L}\|
$$

for all real numbers $\theta_{1}, \ldots, \theta_{m}$ and so part (a) is proved. To prove part (b) note first of all that from the multinomial formula (see [1, p. 38]) we have

$$
\begin{aligned}
& \left(m ! / m^{m}\right)\left|L\left(x_{1}, \ldots, x_{m}\right)\right|=\|\hat{L}\|=\left|\hat{L}\left(\left(\sum_{j=1}^{m} e^{i \theta_{j}} x_{j}\right) / m\right)\right| \\
& =\left(1 / m^{m}\right)\left|\sum\left(m ! / n_{1} ! \cdots n_{m} !\right) L\left(\left(e^{i \theta_{1}} x_{1}\right)^{n_{1}} \cdots\left(e^{i \theta_{m}} x_{m}\right)^{n_{m}}\right)\right|,
\end{aligned}
$$

where the summation is over all $m$-tuples $\left(n_{1}, \ldots, n_{m}\right)$ of nonnegative integers satisfying $n_{1}+\cdots+n_{m}=m$. Since the last equation is true for all real numbers $\theta_{1}, \ldots, \theta_{m}$ we get

$$
\begin{aligned}
& \left(m !\left|L\left(x_{1}, \ldots, x_{m}\right)\right|\right)^{2} \\
& \quad=\int_{T^{m}}\left|\sum\left(m ! / n_{1} ! \cdots n_{m} !\right) L\left(\left(e^{i \theta_{1}} x_{1}\right)^{n_{1}} \cdots\left(e^{i \theta_{m}} x_{m}\right)^{n_{m}}\right)\right|^{2} d \lambda(\theta) .
\end{aligned}
$$

From the above equation it follows that

$$
\sum\left(\left(m ! / n_{1} ! \cdots n_{m} !\right)\left|L\left(x_{1}^{n_{1}} \cdots x_{m}^{n_{m}}\right)\right|\right)^{2}=0
$$

where the summation is over all $m$-tuples $\left(n_{1}, \ldots, n_{m}\right)$ of nonnegative integers, at least one of which is greater than 1 , satisfying $n_{1}+\cdots+n_{m}=m$. This proves part (b).

Since (ii) obviously implies (iii) we have to prove only that (iii) implies (i). But conditions (a), (b) of (iii) and the multinomial formula give us

$$
\|\hat{L}\|=\left(m ! / m^{m}\right)\left|L\left(x_{1}, \ldots, x_{m}\right)\right|
$$


and since by hypothesis we have $\|L\|=\left(\mathrm{m}^{m} / \mathrm{m} !\right)\|\hat{L}\|$, it follows that

$$
\|L\|=\left|L\left(x_{1}, \ldots, x_{m}\right)\right| \text {. }
$$

Working in a similar fashion, it can also be proved that if $E$ is a Banach space and $\left|L\left(x_{1}, \ldots, x_{m}\right)\right|=\|L\|$ for some $L \in \mathscr{L}_{m}^{s}(E, \mathbf{C})$, where $x_{1}, \ldots, x_{m}$ are unit vectors in $E$, then conditions (ii) and (iii) of Theorem $2^{\prime}$ are equivalent to the condition

(i')

$$
\|L\|=\left(m^{m} / m !\right)\|\hat{L}\| .
$$

The following example shows that the converse of Theorem 2 is false, so for equivalent conditions the complications of Theorem 2 ' are necessary.

EXAMPLE. We consider the canonical example (1) in the case $m=3$. Then

$$
\|\Phi\|=\left(3^{3} / 3 !\right)\|\hat{\Phi}\| \text {. }
$$

Also for the unit vectors $x=(1 / 2,1 / 2,0), y=(1 / 2,0,1 / 2), z=(0,1 / 2,1 / 2)$ of $l_{3}^{1}$ we have $\hat{\Phi}(x)=\hat{\Phi}(y)=\hat{\Phi}(z)=0$ and $\hat{\Phi}$ achieves its norm at the point $(1 / 3,1 / 3,1 / 3)$. However $\Phi(x, y, z)=1 / 24<\|\Phi\|$, since $\|\Phi\|=1 / 6$.

Suppose that $L \in \mathscr{L}_{m}^{s}(E, \mathbf{C})$ satisfies (5) and let $F$ be the restriction of $L$ to $B=\operatorname{span}\left\{x_{1}, \ldots, x_{m}\right\}$. Since (7) holds, it follows from the proof of Theorem 1 that for any complex numbers $a_{1}, \ldots, a_{m}$ we have

$$
\left\|a_{1} x_{1}+\cdots+a_{m} x_{m}\right\|=\sum_{k=1}^{m}\left|a_{k}\right| .
$$

Thus $B$ is isometrically isomorphic to $l_{m}^{1}$. Note also that

$$
\left|F\left(x_{1}, \ldots, x_{m}\right)\right|=\left(m^{m} / m !\right)\|\hat{F}\| .
$$

Now since $F$ satisfies condition (ii)(b) we get the following result.

COROLlaRy 1. Let $C(m, E)=m^{m} / m$ ! and $L$ be an extremal continuous symmetric $m$-linear form, which achieves its norm at the point $x=\left(x_{1}, \ldots, x_{m}\right)$ of the unit sphere of $E^{m}$. Then $F=\left.L\right|_{B}$, where $B=\operatorname{span}\left\{x_{1}, \ldots, x_{m}\right\}$ is a continuous symmetric $m$-linear form on $B$, and $F=c \Phi$, where $c$ is a constant and $\Phi \in \mathscr{L}_{m}^{s}\left(l_{m}^{1}, \mathbf{C}\right)$ is given by (1).

If $E$ is an $m$-dimensional Banach space, then every multilinear form on $E$ is continuous. If $L$ is a symmetric $m$-linear form on $E$, then $L$ achieves its norm since the unit ball of $E^{m}$ is compact. Using these remarks we obtain another corollary.

COROllary 2. An m-dimensional Banach space $E$ is isometrically isomorphic to $l_{m}^{1}$ if and only if $C(m, E)=m^{m} / m !$. If $C(m, E)=m^{m} / m !$, then every extremal symmetric $m$-linear form on $E$ is of the form $L=c \Phi$ for some constant $c$ and $\Phi \in \mathscr{L}_{m}^{s}\left(l_{m}^{1}, \mathrm{C}\right)$ defined by (1).

Finally notice that using techniques similar to those of the proof of Theorem 1, we can prove Theorem 1 in the case where $E$ is a real normed space. However for Theorem 2', if $E$ is a real Banach space, we need a different approach. We hope to discuss this in a subsequent paper. 
I would like to thank my research supervisor, Professor A. M. Tonge, for suggesting the problem and for his constant help. I am also grateful to Professor R. M. Aron for his pertinent remarks.

\section{REFERENCES}

1. S. B. Chae, Holomorphy and calculus in normed spaces, Dekker, New York, 1985.

2. L. A. Harris, Bounds on the derivatives of holomorphic functions of vectors, Colloque d'Analyse ( $\mathrm{L}$. Nachbin, ed.), Rio de Janeiro, 1972, Actualités Sci. Indust., no. 1367, Hermann, Paris, 1975, pp. 145-163.

3. J. Lindenstrauss and L. Tzafriri, Classical Banach spaces. II. Function spaces, Ergebnisse der Math., Band 97, Springer, 1979.

4. R. S. Martin, Thesis, California Inst. of Tech., 1932.

5. I. Sarantopoulos, Estimates for polynomial norms on $L^{p}(\mu)$-spaces, Math. Proc. Cambridge Philos. Soc. 99 (1986), 263-271.

6. Seminaire Maurey-Schwartz, Exposé VII, 1973-74.

7. A. M. Tonge, Polarization and the complex Grothendieck inequality, Math. Proc. Cambridge Philos. Soc. 95 (1984), 313-318.

8. The Scottish Book (Mathematics from the Scottish Café) (R. D. Mauldin, ed.), Birkhaüser, Boston, Mass., 1981.

Department of Mathematical Sciences, Kent State University, Kent, Ohio 44242

Current address: Department of Mathematics, National Technical University of Athens, Zografou Campus, 15773 Athens, Greece 